\title{
DEVELOPMENT OF THE STRUCTURE OF INTELLECTUAL AUTOMATIC CONTROL SYSTEM
}

\author{
— short communcation - \\ G.V. ABRAMOV ${ }^{1}$, Ivan V. ZHELTOUKHOV ${ }^{1}$ \\ FSBEI HPE Voronezh State University of Engineering Technologies, \\ Voronezh, Russia
}

\begin{abstract}
Relevance of the study is associated with reduced energy intensity of the process heat demand by improving the systems and control algorithms of automatic energy management in buildings. This paper presents a system for power management of individual modules with interaction via the Ethernet, allowing measuring the qualitative and quantitative performance of network communication protocols of different levels of performance monitoring in real time. The difference of the developed intellectual automatic control system consisting of separate modules with interaction via the Ethernet, there is no separation devices for simple and intelligent - all the devices are intelligent, the controller is a separate module, and its functions are performed by the control unit.
\end{abstract}

Keywords: automatic control system, the algorithm, the distribution of the time of delivery, energy consumption, energy saving

\section{INTRODUCTION}

Energy-saving technologies in recent years have become more in demand. For Russian energy conservation issues are of particular relevance, since climatic conditions fuel costs on people's warmth in Russia are the highest.

1 Corresponding authors. Mailing adress: FSBEI HPE Voronezh State University of Engineering Technologies, Voronezh, Russia (394036, Voronezh, Revolution Avenue, 19), e-mail: webster2580@gmail.com

Vol. XVII (2013), no.2 
Energy-saving technologies have become more actively developed and implemented after the oil crisis of the 1970s, which changed radically the global energy mentality. It is more efficient use of energy, not its production capacity, it has become the main means of overcoming the energy crisis (Tabunschikov, 2010).

Thus, the relevance of energy efficiency in Russia is determined by the folyuschim:

- climatic location of the country;

- gradual depletion of stocks, the increasing complexity of production and an increase in the cost of natural fossil fuels;

- increasing in energy consumption;

- wasteful power consumption;

- complication of environmental conditions associated with increased emissions of toxic and carcinogenic products of combustion.

The modern systems of technological processes on the lower control level using network technologies. Recently, there has been a trend towards data transmission technology Ethernet (Abramov et al., 2010). The disadvantages of this technology are competing access, queues, collision. The lower level of the control system is necessary to ensure the delivery of information in a given time, is now Ethernet technology does not guarantee it.

System structure of the automatic control system with interaction via an Ethernet network includes sending data using the protocol UDP, performing configuration of network elements, sending the first broadcast data packet synchronization of time in relation to receiving the first data packet to determine the correct operation of the device by sending packets at regular intervals, with each device may send data to any other device on the network data stored in the control law controller and the controller can work with several control loops.

\section{MATERIALS AND METHODS}

The proposed intelligent automatic control system of separate modules with interaction via Ethernet devices there is no separation into simple and intelligent - all the devices are intelligent, the controller is a separate module, and its functions are performed by the control unit (Abramov and Zheltoukhov, 2012a). Such a structure makes it possible to facilitate the configuration of the network, reduce the number of broadcast packets and reduce the overall system cost (Abramov and Zheltoukhov, 2012b) .

Vol. XVII (2013), no.2 
For intelligent automatic control algorithms were developed following actions:

- algorithm for connection of the controls;

- algorithm for sensor connection;

- algorithm update of the devices;

- algorithm System Health Validator.

Algorithm for connecting the device consists of the following steps:

1. Issuance of the device, the identifier (IP-addresses) for subsequent check of the devices in the network. Testing is done by SNMP protocol or ICP.

2. Check for re-adding the device, you need to avoid situations of multiple registration of one device in the system. If upon examination found the registered device with an identifier, the system proposes to update the data on a connected device.

3. Setting up a root user, after receiving the device ID, the following:

- unique device name (string);

- unit for actual values of the device (string);

- lower and upper limits for actual value (two real values).

4. Adding a new device to a database of registered devices.

To check the availability of the device in the network uses the SNMP. This allows you to get information about the device, from which can then be necessary to remove the tuning parameters, so is preferred to c report ICP, which will only detect the connected device.

Algorithm for connecting the device which combines the control unit and the sensor consists of the following, partly overlapping with the sequences to connect the control unit and the sensor steps :

1. Issuance of the device, the identifier (IP-addresses ) for subsequent check of the devices in the network. Testing is done by SNMP protocol or ICP.

2. Check for re-adding the device, you need to avoid situations of multiple registration of one device in the system. If upon examination found the registered device with an identifier, the system proposes to update the data on a connected device.

3. Setting up a root user, after receiving the device ID, the following:

- unique device name (string);

- unit for actual values of the device (string) ;

- lower and upper limits for actual value .

- weight coefficient recordable data reading device.

5. Adding a new device to a database of registered devices.

Algorithm for connecting the sensor consists of the following steps :

Acta Universitatis Cibiniensis Series E: FOOD TECHNOLOGY

Vol. XVII (2013), no.2 
1. Issuance of the device, the identifier (IP-addresses ) for subsequent check of the devices in the network.

2. Check for re-adding the device, you need to avoid situations of multiple registration of one device in the system. If upon examination found the registered device with an identifier, the system proposes to update the data on a connected device.

3. Setting up a root user, after receiving the device ID, the following:

- unique device name (string);

- the units of the measured values of the device (string);

- Lower and upper limits of the measurement of the measured value ;

- weight coefficient recordable data reading device.

4. Adding a new device to a database of registered devices.

Updating of the network devices is performed as in the case of physical removal of a device from the system, and further connecting the other with the same identifier. How to update the data on the devices in the system includes the following steps :

a) Selection of the presented device IDs whose data should be updated .

b) Give a new class to which this device .

c) Specify all the required settings of the device .

d) Updating the data on the device in the database of registered devices .

The algorithm SHV contains the following steps :

- Ping a server archive storage in the absence of the designated prompts the user for permission to work without saving history.

- Checking communication with all devices ( control units, sensors ) that are registered in the system.

- When the communication with the archive repository is made to establish a connection with him and fetch relevant data necessary to initiate the process of governance.

\section{CONCLUSIONS}

The above method allows you to keep track of the accident, and allows a single device -operation monitoring network to work with multiple sensors. The proposed method of building intelligent automatic control system with interaction via Ethernet allows you to:

- simplify network configuration by storing control data in the controller;

- reduce the load on the network due to the fact that broadcast packets are sent only when registering for device monitoring network, and the work is

Vol. XVII (2013), no.2 
carried out only between devices on IP-addresses, without additional identifiers;

- reduce the cost of the system due to the absence in the system of a single device controller..

\section{REFERENCES}

1. Tabunschikov, Y.A. (2010) Energy efficient buildings - the possibilities of the Moscow construction. Moscow: Power Saver.

2. Abramov, G.V, Emelyanov A.E, Ryazanov A.N, Ivashin, A.L. (2010). The validity of the use of Ethernet in industrial distributed control systems. FPS Finance. Economy. Strategy, Voronezh, Russia.

3. Abramov, G.V.; Zheltoukhov I.V. (2012a) System structure of the automatic control. Collection of scientific papers based on the I International Scientific and Practical Internet Conference "Modelling of Energy and Process", Voronezh, Russia.

4. Abramov, G.V.; Zheltoukhov I.V. (2012b) Intellectual Development of automatic control system. Collection of scientific papers based on the XIV International Scientific and Technical Conference "Science and High Technology of XXI century", Voronezh, Russia.

Vol. XVII (2013), no.2 